\title{
Neutral and charged embedded clusters of Mn in doped GaN from first principles
}

\author{
X. Y. Cui, ${ }^{1}$ B. Delley, ${ }^{2}$ A. J. Freeman, ${ }^{3}$ and C. Stampfl ${ }^{1}$ \\ ${ }^{1}$ School of Physics, The University of Sydney, Sydney 2006, New South Wales, Australia \\ ${ }^{2}$ Paul Scherrer Institut, WHGA/123, CH-5232 Villigen PSI, Switzerland \\ ${ }^{3}$ Department of Physics and Astronomy, Northwestern University, Evanston, Illinois 60208-3112, USA
}

(Received 25 February 2007; published 2 July 2007)

\begin{abstract}
Based on extensive density-functional theory calculations, the spatial distribution and magnetic coupling of $\mathrm{Mn}$ atoms in Mn:GaN have been reinvestigated by doping up to five Mn atoms in large supercells, where the formation energies and the electronic structure for both the neutral and charged valence states are studied. The doped $\mathrm{Mn}$ atoms have a strong tendency to form substitutional Mn-N-Mn bonded embedded clusters with short-range magnetic interactions, where the long-range wurtzite structure is maintained. While for neutral pair doping the coupling is ferromagnetic regardless of the distance and orientation of the $\mathrm{Mn}$ atoms, the negatively charged states tend to weaken the parallel coupling. Significantly, for larger (than pair) cluster configurations for both neutral and all the energetically favorable charged states, states containing antiparallel coupling are always favored. Thus, we argue that the "giant cluster moment" in Mn:GaN, as proposed by Rao and Jena based on study of free clusters [Phys. Rev. Lett. 89, 185504 (2002)] and calculated by Sandratskii et al. [Phys. Rev. B 71, 045210 (2005)], is not applicable for "larger" (>2) Mn-cluster-doped GaN. The size of the supercells employed and the atomic relaxation are found crucial for an accurate description, and are partially responsible for these discrepancies. Also important is that the electrical conductivity of $\mathrm{Mn}: \mathrm{GaN}$ depends sensitively on the valence states, where the negatively charged state $\operatorname{Mn}^{2+}\left(d^{5}\right)$ exhibits highly insulating character as observed in experiments. The formation of embedded clustering leads to a strong local structural distortion and a significant spin polarization on neighboring $\mathrm{N}$ atoms due to hybridization of $\mathrm{Mn} 3 d$ and $\mathrm{N} 2 p$ orbitals. Our results highlight the intrinsically complex nature of the spatial distribution and magnetism in transition metal doped dilute magnetic semiconductors, and can rationalize some hitherto puzzling experimental observations, notably the low saturation moments, the contracted lattice constants, and the highly insulating behavior.
\end{abstract}

DOI: $10.1103 /$ PhysRevB.76.045201

PACS number(s): 71.23.-k, 71.55.Eq, 75.50.-y, 85.75.-d

\section{INTRODUCTION}

Since the discovery of the carrier-induced ferromagnetism in Mn:InAs (Ref. 1) and Mn:GaAs (Ref. 2), III-V-type diluted magnetic semiconductors (DMSs) have attracted intense attention in view of their potential as spintronic devices. It is widely expected that new functionalities of electronics and photonics can be derived if the injection, transfer, and detection of carrier spin can be controlled above room temperature in DMSs. ${ }^{3,4}$ Traditional III-V DMSs, such as Mn:GaAs, suffer from the limitation of the Curie temperature $\left(T_{C}\right)$ to cryogenic temperature $(\sim 170 \mathrm{~K})$, which significantly circumscribes their practical use in room temperature devices.

Room temperature ferromagnetism in transition metal (TM)-doped III-nitrides may provide a good opportunity for the integration of spintronics with existing III-nitride based photonic and electronic devices. Quite recently, many groups have focused on GaN-based DMS. One of the reasons is the relatively long electron spin lifetime due to the reduced spinorbit coupling, in comparison with GaAs. ${ }^{5,6}$ An additional motivation is the prediction of room temperature ferromagnetism in Mn:GaN by Dietl et al. ${ }^{7}$ on the basis of a meanfield Zener model. Although the Mn level is predicted to lie deep in the gap in cubic $(\mathrm{Ga}, \mathrm{Mn}) \mathrm{N}$, namely, $1.42 \mathrm{eV}$ (Refs. 8 and 9) above the valence-band maximum by optical absorption spectroscopy, it has been demonstrated that it can be highly $p$ type, with carrier concentrations exceeding
$10^{18} \mathrm{~cm}^{-3}$ at room temperature; $;^{10}$ it therefore has potential for hole-mediated ferromagnetism, as found e.g., in Mn:GaAs.

While the origin and the mechanism of DMS are still currently under debate, Mn:GaN based systems present a particularly controversial subject. Mn-doped GaN thin films have been grown successfully using a variety of techniques. However, so far experimental results appear strongly scattered; magnetic ground states reported to range from paramagnetic ${ }^{11}$ to ferromagnetic (FM), with $T_{C}$ of 10 and $25 \mathrm{~K},{ }^{12} 288-370 \mathrm{~K}^{13}$ and $940 \mathrm{~K},{ }^{14-17}$ to spin glass states. ${ }^{18,19}$ In particular, Sonoda and co-workers ${ }^{16,17}$ grew wurtzite Mn-doped GaN films by molecular-beam epitaxy (MBE) and magnetic measurements showed a very high $T_{C}$ of about $940 \mathrm{~K}$. They also ruled out the possibility of phase segregation of some FM compounds, e.g., $\mathrm{MnGa}$ and $\mathrm{Mn}_{4} \mathrm{~N}$ which also have high $T_{C}$. Instead, they proposed a double exchange model for the high $T_{C}$ ferromagnetism in Mn:GaN. ${ }^{17}$ Alternatively, some experimental results show antiferromagnetic (AFM) coupling between Mn ions at $T$ $=2 \mathrm{~K}$ (Refs. 20-22) and others show a FM phase with the existence of secondary phases ${ }^{23-27}$ or without secondary phases ${ }^{12,13,16,28-32}$ within the detection limits. Very recently, the intrinsic ferromagnetism in wurtzite $\mathrm{Mn}: \mathrm{GaN}$ was demonstrated with a rather large magnetic moment of 2.4 $\mu_{B} / \mathrm{Mn}$, but the $T_{C}$ is only about $8 \mathrm{~K}$ for the concentration of $6.8 \%$ sample. ${ }^{33}$ Clearly, the growth methods and conditions, as well as the characterization, are important factors regarding these discrepancies. For example, implanted high energy 
Mn ions will seriously damage the crystal structure so that it is impossible to restore by postannealing; consequently, the formation of secondary phases is inevitable. ${ }^{34}$ Similar effects are expected for the crystals grown by ammonothermal and chemical transport methods. ${ }^{28}$ Experimentally, given the level of dilution of the magnetic ions (typically a few percent), it is often very difficult to categorically determine the spatial distribution and the magnetic coupling of the dopants.

On the theoretical side, the majority of first-principles investigations into $\mathrm{Mn}: \mathrm{GaN}$ using the supercell approach have focused on "single" $18,35-40$ atom doping on the cation sites, where the change of concentration is achieved by varying the size of the supercells. This is based on the assumption of (i) a homogeneous distribution and (ii) a FM coupling between the periodic neighboring supercells. However, the critical dependence of the magnetic properties on sample growth details, particularly the doping concentration, clearly suggests that such a description is incomplete. A "pair-doping" model (doping two $\mathrm{Mn}$ on cation sites in supercells and comparing the stability of the FM and AFM states to decide the ground state) has been widely used to investigate the tendency of clustering and the magnetic coupling, ${ }^{39,41-46}$ where it is predicted that FM is favored over the AFM state. ${ }^{39,41,44-46}$ Recently, Sanyal and Mirbt reported AFM alignment between substitutional $\mathrm{Mn}$ and interstitial $\mathrm{Mn}$ in $\mathrm{GaN} .{ }^{47}$ Interestingly, Wang et al. ${ }^{48}$ demonstrated that $\mathrm{Mn}$ atom pairs, which couple ferromagnetically in bulk Mn:GaN, couple antiferromagnetically near the surface due to bond length contraction. Conceptually, this pair-doping model might be invalid or incomplete as a third or more magnetic dopant atoms may spoil this simplistic description. We recently demonstrated this for $\mathrm{Cr}: \mathrm{GaN}$ systems, ${ }^{49,50}$ where the stability and magnetic properties of $\mathrm{Cr}$ atoms are fundamentally altered by the presence of the additional $\mathrm{Cr}$ atoms, showing that a "polydoping" (in contrast to a pair-doping) study is indeed crucial to give a correct description regarding the magnetic interactions.

In this respect, a systematic theoretical polydoping investigation in $\mathrm{Mn}: \mathrm{GaN}$ is a tantalizing subject, particularly in view of some recent experimental advances. Since the equilibrium solubility of TM atoms is very low, III-nitride DMS thin film samples have to be grown under nonequilibrium conditions and the doped magnetic ions are rather mobile at elevated temperature, and consequently clustering in DMS is feasible. Indeed, experiment confirmed the diffusion of $\mathrm{Mn}$ atoms into the GaN to a depth of $380 \AA .{ }^{13} \mathrm{X}$-ray absorption near-edge structure spectra have suggested the presence of short-range disorder, while the long-range crystalline order was retained. Moreover, it has been reported that Mn atoms are "somewhat interrelated among themselves," and that they are not randomly distributed in the GaN lattice. ${ }^{51}$ It was intuitively suggested that the observed arrangement of Mn atoms might be due to the formation of "Mn clusters." ${ }^{51}$ In addition, the formation of metallic Mn-rich clusters in GaN has also been indicated by the highly accurate synchrotron radiation microprobe. ${ }^{52}$ It was shown that preservation of the hexagonal symmetry in both cluster-free and Mn-rich regions and preferential disordered cluster formation were observed in the direction parallel to the crystal growth (along the [001] direction) rather than perpendicular to it. ${ }^{53}$ More directly, structural characterization by various techniques re- veals that a $\mathrm{Mn}: \mathrm{GaN}$ sample with a Mn content of $13.7 \%$ exhibits nanometer-sized Mn-rich clusters which are embedded in the $(\mathrm{Ga}, \mathrm{Mn}) \mathrm{N}$ alloy and are coherent with the surrounding matrix. ${ }^{24}$

Several first-principles theoretical investigations into clustering in Mn:GaN have been carried out. In particular, van Schilfgaarde and Mryasov ${ }^{54}$ first suggested that in TM-doped III-V semiconductors, the magnetic impurities may aggregate into "small nanoclusters of a few atoms." However, no systematic search for the atomic and magnetic ground state was carried out at that time. Subsequently, for $\mathrm{Cr}$ and $\mathrm{Mn}$ in GaAs and GaN, taking into account the magnetic atom pair interaction, Xu et al. ${ }^{55}$ concluded that disorder (and clustering) reduces the critical temperature, while ordering of the dopants increases it. Alternatively, Rao and Jena ${ }^{56}$ investigated the stability and magnetic properties of small Mn gas phase clusters and found that they can be fundamentally altered by the presence of a nitrogen atom; it was thus suggested that the formation of small $\mathrm{Mn}_{x} \mathrm{~N}(x=1-5)$ clusters carrying "giant magnetic moments" $\left(4-4.5 \mu_{B} / \mathrm{Mn}\right)$ might be responsible for the high $T_{C}$ detected in some Mn:GaN samples. Subsequently, Sandratskii et al. ${ }^{57}$ reported an investigation of Mn clustering in $\mathrm{GaN}$ by doping up to $4 \mathrm{Mn}$ atoms in a 64-atom supercell without atomic relaxation. While their results "confirmed" the formation of the giant cluster moments, with values around $3.4 \mu_{B} / \mathrm{Mn}$, they showed, however, that the $T_{C}$ decreases with the formation of these small clusters. Very recently, based on densityfunctional theory (DFT) together with the mean-field approximation, Hynninen et al. ${ }^{58}$ predicted that $T_{C}$ depends crucially on the microscopic cluster distribution. For example, the partially dimerized pair-Mn-single-Mn complex distribution is found to give the highest $T_{C}>500 \mathrm{~K}$, while the lowest $T_{C}$ values (close to zero) are obtained for the quarto-Mn-single-Mn and quarto-Mn-trio-Mn distributions. Note that in this study, the magnetic interaction within the Mn clusters was assumed to be FM. In the present paper, importantly, we show that the giant moments may actually not be expected, or it strongly depends on the doping concentration; when using larger supercells and fully relaxed atomic structures, the AFM state is found to be more energetically favorable, resulting in partial cancellation of atomic moments and a notably reduced net spin.

Another motivation for this study is that despite several theoretical investigations which predict the appealing halfmetallicity in Mn:GaN systems, ${ }^{35,39,41,44,47,59}$ the experimental results show that they are highly resistive ${ }^{22}$ and even semi-insulating ${ }^{60}$ or insulating. ${ }^{19,24}$ The explanation for this outstanding contradiction is unknown. Furthermore, despite experimental evidence of the coexistence of negatively charged $\mathrm{Mn}^{2+}\left(d^{5}\right)$ and neutral $\mathrm{Mn}^{3+}$ ions in $\mathrm{Mn}: \mathrm{GaN}^{6}{ }^{61-63}$ the majority of theoretical studies have hitherto been limited to neutral systems, i.e., the $\mathrm{Mn}^{3+}\left(d^{4}\right)$ ion. The possible important effects of charged states on the magnetic and electronic properties are largely unexplored. Hence, a systematic study of charged state and the effect on the properties is highly desirable.

Clearly, knowledge of the spatial distribution and the magnetic coupling between magnetic dopants is a prerequisite to understanding the mechanisms of DMS and estimat- 
ing the critical temperature. In this paper, we present a comprehensive theoretical investigation using DFT and evaluate the relative stability of various doping configurations in large supercells, including various single-doping, substitutional clusters, substitutional-interstitial complexes, and also Mn-Mn bonded configurations. We first perform a comprehensive investigation for the neutral state and then, for the most stable structures, calculate various charged states. We find that the charged states are vitally important for the magnetic coupling and the electrical conductivity. We also address the importance of the supercell size and the atomic relaxation in $\mathrm{Mn}: \mathrm{GaN}$. We propose a picture that various cluster configurations (perhaps with different valence states) embedded in the host wurtzite semiconductor coexist, which depend sensitively on the growth conditions. On the basis of our theoretical results, we then rationalize some puzzling experimental observations.

\section{CALCULATION DETAILS}

We perform DFT calculations using the generalized gradient approximation ${ }^{64}$ with the periodic DMOL ${ }^{3}$ code $^{65}$ (Academic Version 3.9), where the density-functional semilocal pseudopotentials ${ }^{66}$ are used. In this scheme, all electrons for $\mathrm{N}$ are included, while for $\mathrm{Ga}$ and $\mathrm{Mn}$ the $3 d^{10} 4 s^{2} 4 p^{1}$ and $3 s^{2} 3 p^{6} 3 d^{5} 4 s^{2}$ electrons are treated as valence electrons, respectively. A double set of numerical valence functions with a local basis cutoff $R_{c}$ of 11.0 a.u. is used. The optimized wurtzite lattice constants are $a=3.184 \AA$ and $c=5.184 \AA$ with internal parameter $u=0.3765$, which are in good agreement with experimental and previous theoretical results. ${ }^{67}$ Various supercells, 32-atom $(2 a \times 2 a \times 2 c)$, 72-atom (3a $\times 3 a \times 2 c)$, 96-atom (cubic) $(2 \sqrt{3} a \times 3 a \times 2 c), 108$-atom $(3 a \times 3 a \times 3 c), 128$-atom $(4 a \times 4 a \times 2 c)$, and 256-atom ( $4 a$ $\times 4 a \times 4 c$ ), are used. Large supercells ensure that for each configuration studied, the interactions between neighboring supercells are negligible. Convergence as a function of supercell size has been checked for all the favorable configurations. We find that the size of the supercell is crucial in determining the correct magnetic coupling and electronic structure. Reciprocal space k-point meshes of $8 \times 8 \times 6$ for the 32-atom, $4 \times 4 \times 3$ for the 72 -atom, and $2 \times 2 \times 2$ for the 96-, 108-, and 128-atom supercells and $2 \times 2 \times 1$ for the 256 atom cell are employed. Except where specified, atomic relaxation of all atoms is included for all the calculations.

We calculate the formation energy for the neutral and charged states to determine the relative stability of the different configurations, ${ }^{68}$ for which the formation energy for a charged state $q$ for a given configuration is calculated as

$$
\begin{aligned}
E^{f}= & E_{\mathrm{Mn}: \mathrm{GaN}}-E_{\mathrm{ref}-\mathrm{GaN}}-n \mu_{\mathrm{Mn}}+m \mu_{\mathrm{Ga}}+l \mu_{\mathrm{N}} \\
& +q\left(E_{F}+E_{v}+\Delta V\right),
\end{aligned}
$$

where $E_{\mathrm{Mn}: \mathrm{GaN}}$ and $E_{\text {ref-GaN }}$ are the total energies of $\mathrm{Mn}$ doped $\mathrm{GaN}$ and the pure $\mathrm{GaN}$ reference structure (as calculated with the same size supercell), respectively. Here, $\mu_{\mathrm{Ga}}$, $\mu_{\mathrm{Mn}}$, and $\mu_{\mathrm{N}}$ are the atom chemical potentials of $\mathrm{Ga}, \mathrm{Cr}$, and $\mathrm{N}$, while the integers $n, m$, and $l$ are the numbers of doped $\mathrm{Mn}$ atoms and substituted $\mathrm{Ga}$ and $\mathrm{N}$ atoms, respectively. $E_{F}$ is the Fermi level, referenced to the valence-band maximum in bulk $\mathrm{GaN}$, and $E_{v}$ is the bulk valence-band maximum of the reference supercell. The correction term $\Delta V$ is used to align the reference electrostatic potential between the supercell containing the charged defect and the bulk supercell. In this work, this potential shift is determined by comparing the values of the $\mathrm{N} 1 s$ core level. The implementation of the charged state feature in $\mathrm{DMOL}^{3}$ is based on Janak's theorem. ${ }^{69}$ The chemical potentials depend on the experimental conditions under which the material is grown. In order to determine these quantities, we invoke the relationship $\mu_{\mathrm{Ga}}+\mu_{\mathrm{N}}$ $=\mu_{\mathrm{GaN}}$, assuming both species are in thermal equilibrium with GaN. Furthermore, the chemical potentials must satisfy the boundary conditions $\mu_{\mathrm{N}}<1 / 2 \mu_{\mathrm{N}_{2}}$ and $\mu_{\mathrm{Ga}}<\mu_{\mathrm{Ga} \text { (bulk) }}$ (if this were not the case, then GaN would be thermodynamically unstable with respect to the formation of $\mathrm{N}_{2}$ molecules or bulk Ga). When imposing certain growth conditions [nitrogen rich $\left(\mu_{\mathrm{N}}=1 / 2 \mu_{\mathrm{N}_{2}}\right)$ or gallium rich $\left.\left(\mu_{\mathrm{Ga}}=\mu_{\mathrm{Ga} \text { (bulk) }}\right)\right]$, the chemical potential for the other species can be determined from the above (thermal equilibrium) relationship. Under nitrogen-rich conditions, $\mu_{\mathrm{Ga}}=E_{\mathrm{GaN}(\mathrm{bulk})}-1 / 2 E_{\mathrm{N}_{2}}$, and under gallium-rich conditions, $\mu_{\mathrm{N}}=E_{\mathrm{GaN}(\mathrm{bulk})}-E_{\mathrm{Ga}(\mathrm{bulk})}$. The atomic chemical potential for $\mathrm{Mn}$ is evaluated by assuming thermal equilibrium with rocksalt $\mathrm{MnN}$ (Ref. 70) and $\mathrm{CuAu}-$ type GaMn (Ref. 71) under N-rich and Ga-rich conditions, respectively, whereby the chemical potential is lowest (leading to maximum solubility). Then, under $\mathrm{N}$-rich conditions, $\mu_{\mathrm{Mn}}=E_{\mathrm{MnN}(\text { bulk })}-1 / 2 E_{\mathrm{N}_{2}}$, and under Ga-rich conditions, $\mu_{\mathrm{Mn}}=E_{\mathrm{GaMn}(\mathrm{bulk})}-E_{\mathrm{Ga}(\text { bulk) }}$. These values for $\mu_{\mathrm{Mn}}$ are more stable than those obtained from bulk $\alpha$-Mn (Ref. 72) by 0.86 and $0.40 \mathrm{eV} / \mathrm{Mn}$, respectively. The calculated heat of formation of $\mathrm{GaN}$ is $-1.04 \mathrm{eV}$, which is in good agreement with the established data (experimental value is $-1.17 \mathrm{eV}$ ). ${ }^{68}$ The calculated structural parameters and energetic data for bulk $\mathrm{Ga}$ and the $\mathrm{N}_{2}$ molecule are reported elsewhere. ${ }^{50}$

Considering that to our knowledge there are no reported formation energies for charged states in TM-doped DMS systems, we have rigorously checked that we obtain consistent results for well established charged native defects, namely, a $\mathrm{N}$ vacancy in AlN and a Ga vacancy in GaN. For the former system, excellent agreement with previous results was obtained. ${ }^{73}$ For the latter, the shape of the formation energy curve (comparing the neutral, $-1,-2$, and -3 charged states) is well reproduced. However, our calculated formation energies are systematically lower than the reported values in Refs. 68 and 74, e.g., $7.6 \mathrm{eV}$ versus $9.1 \mathrm{eV}$ for the neutral state. This discrepancy could be due (i) to the treatment of the Ga $3 d$ electrons (Ref. 74 used norm-conserving pseudopotentials with the nonlinear core correction for the Ga $3 d$ electrons, while in the present results, Ga $3 d$ states are explicitly treated as valence electrons) and (ii) to the fact that, in agreement with Ref. 46, we find that the Ga vacancy in $\mathrm{GaN}$ actually represents a native magnetic system and the spin-polarization effect, for example, giving an energy gain of $0.34 \mathrm{eV}$ for the neutral systems as calculated in the 96atom cell, was not considered in Ref. 74. 
(a)
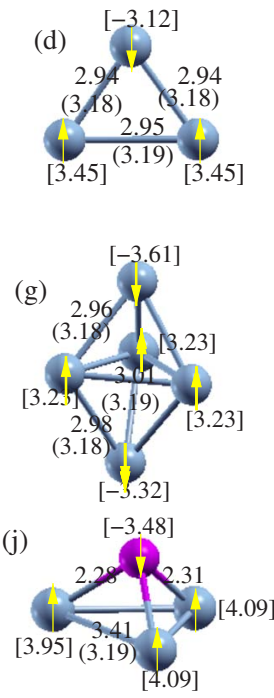

(h)
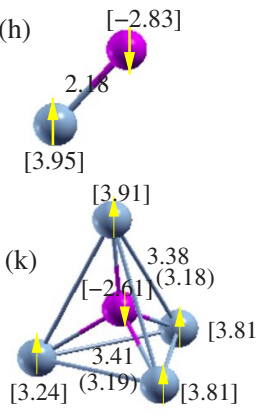

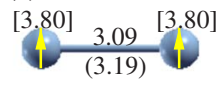

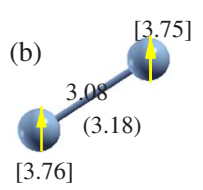

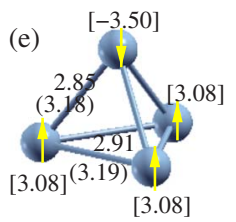

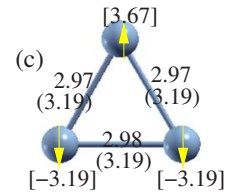

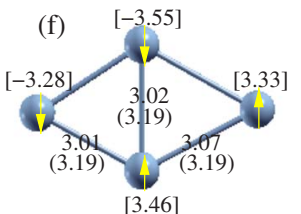

(i)

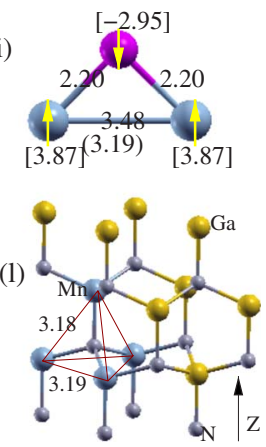

FIG. 1. (Color online) Optimized Mn clustering geometries and magnetic structures. The blue circles represent substitutional $\mathrm{Mn}_{\mathrm{Ga}}$ and the pink interstitial $\mathrm{Mn}_{i-O}$ in (h)-(k). The $\mathrm{Mn}-\mathrm{Mn}$ distances before (in parentheses) and after relaxation are in $\AA$, and the arrows indicate the directions of the local atomic spin for the magnetic ground state. The atomic spin moment values (in square brackets) are in $\mu_{B}$. Note that the links between $\mathrm{Mn}_{\mathrm{Ga}}$ atoms are only a guide for the eye since in the supercells the $\mathrm{Mn}$ atoms are, of course, bonded to $\mathrm{N}$ and $\mathrm{Ga}$ atoms, which are omitted in (a)-(k). [See (1) showing the (e) cluster doping in the "real" GaN supercell.]

\section{RESULTS}

\section{A. Neutral states}

A systematic polydoping study of DMS inevitably involves a large number of geometrical configurations and possible magnetic states, as well as different large supercells. For doping of up to five $\mathrm{Mn}$ atoms, we perform an exhaustive search for the stable structures and magnetic ground states. For a given structure containing $n \mathrm{Mn}$ atoms, we investigate all the $\left(2^{n-1}+1\right)$ spin configurations. For example, for $n=3$, we consider nonmagnetic, FM, $(\uparrow \uparrow \uparrow)$ and three AFM or ferrimagnetic states $(\downarrow \uparrow \uparrow, \uparrow \uparrow \downarrow$, and $\downarrow \uparrow \downarrow)$ by setting different initial spin configurations on the Mn atoms. In the following, "AFM" also includes ferrimagnetism. The energetically favorable configurations are depicted in Fig. 1 and the associated spin moments, formation energies, and magnetic ground states are listed in Table I.

We first consider single doping of $\mathrm{Mn}$ in $\mathrm{GaN}$. Four highsymmetry sites including two substitutional $\left(\mathrm{Mn}_{\mathrm{Ga}}\right.$ and $\left.\mathrm{Mn}_{\mathrm{N}}\right)$ and two interstitial sites (tetrahedral $T, \mathrm{Mn}_{i-T}$ and octahedral $\left.O, \mathrm{Mn}_{i-O}\right)$ are considered. The calculated local moment for $\mathrm{Mn}_{\mathrm{Ga}}$ is $3.76 \mu_{B}\left(4.0 \mu_{B} /\right.$ cell $)$, which is in agreement with previous calculations. ${ }^{40,41,75}$ For $\mathrm{Mn}_{\mathrm{N}}$, a moment of 2.43 $\mu_{B} / \mathrm{Mn}$ is obtained. For $\mathrm{Mn}_{i-O}$ and $\mathrm{Mn}_{i-T}$, the obtained atomic moments are $2.81 \mu_{B}$ and $0.73 \mu_{B}$, respectively,
TABLE I. Formation energies in the neutral state (under N-rich and Ga-rich conditions), net spin values, and ground magnetic states for the various configurations as calculated with the 96-atom cell. The quantities in boldface are for the lowest energy structure.

\begin{tabular}{|c|c|c|c|c|c|}
\hline Structure & $\begin{array}{l}\text { No. of Mn } \\
\text { atoms }\end{array}$ & $\begin{array}{c}E_{\mathrm{N}-\text { rich }}^{f} \\
(\mathrm{eV} / \mathrm{Mn})\end{array}$ & $\begin{array}{c}E_{\mathrm{Ga}-\text {-rich }}^{f} \\
(\mathrm{eV} / \mathrm{Mn})\end{array}$ & $\begin{array}{l}\text { Net spin } \\
\left(\mu_{B} / \mathrm{Mn}\right)\end{array}$ & $\begin{array}{l}\text { Ground } \\
\text { state }\end{array}$ \\
\hline \multicolumn{6}{|c|}{ Single-doping configurations } \\
\hline $\mathrm{Mn}_{\mathrm{Ga}}$ & 1 & 2.59 & 3.17 & 3.76 & \\
\hline $\mathrm{Mn}_{\mathrm{N}}$ & 1 & 9.42 & 7.92 & 2.43 & \\
\hline $\mathrm{Mn}_{i-O}$ & 1 & 7.98 & 7.52 & 2.81 & \\
\hline $\mathrm{Mn}_{i-T}$ & 1 & 9.33 & 8.87 & 0.73 & \\
\hline \multicolumn{6}{|c|}{ Mn-Mn bonded configurations } \\
\hline I & 2 & 5.75 & 5.29 & 1.84 & FM \\
\hline II & 2 & 5.37 & 4.91 & 0.50 & AFM \\
\hline III & 2 & 4.28 & 4.44 & 4.21 & FM \\
\hline IV & 2 & 4.20 & 4.36 & 0.15 & AFM \\
\hline \multicolumn{6}{|c|}{ Substitutional configurations } \\
\hline $\mathbf{a}$ & 2 & 2.33 & 2.91 & 3.81 & FM \\
\hline b & 2 & 2.39 & 2.97 & 3.78 & FM \\
\hline c & 3 & 2.22 & 2.80 & 0.91 & AFM \\
\hline d & 3 & 2.24 & 2.82 & 0.92 & AFM \\
\hline $\mathbf{e}$ & 4 & 1.97 & 2.55 & 1.43 & AFM \\
\hline f & 4 & 2.01 & 2.59 & 0.04 & AFM \\
\hline g & 5 & 2.04 & 2.62 & 0.75 & AFM \\
\hline \multicolumn{6}{|c|}{ Substitutional-interstitial complexes } \\
\hline h & 2 & 4.14 & 4.08 & 0.56 & AFM \\
\hline i & 3 & 3.43 & 3.20 & 1.59 & AFM \\
\hline j & 4 & 3.24 & 2.96 & 2.13 & $\mathrm{AFM}$ \\
\hline k & 5 & 3.35 & 2.98 & 2.43 & AFM \\
\hline
\end{tabular}

where the former value agrees well with that of $2.6 \mu_{B}$ (in the muffin-tin sphere) as obtained by the linear muffin-tin orbital method. ${ }^{40}$ The smaller atomic moments at interstitial sites can be attributed to the compressed electron motion. ${ }^{76}$ As expected, and in agreement with experiments, ${ }^{77-79}$ the $\mathrm{Mn}_{\mathrm{Ga}}$ site is much more favorable by $\sim 5-7 \mathrm{eV} / \mathrm{Mn}$ than the other three configurations. For interstitial doping, the $\mathrm{Mn}_{i-O}$ site is lower in energy than the $\mathrm{Mn}_{i-T}$ site, by $1.23 \mathrm{eV}$ (32atom cell) and $1.34 \mathrm{eV}$ (72-atom cell), consistent with the larger free space for $\mathrm{Mn}_{i-O}(2.2 \AA$ in diameter $)$ as compared to that in $\mathrm{Mn}_{i-T}(1.3 \AA)$. For the neutral single $\mathrm{Mn}_{\mathrm{Ga}}$, besides the high spin ground state of $4.0 \mu_{B} /$ cell, a metastable nonmagnetic state is also predicted. ${ }^{80,81}$ Overall, based on the calculated formation energies, we predict that isolated interstitial $\mathrm{Mn}$ and $\mathrm{Mn}_{\mathrm{N}}$ are highly unlikely in $\mathrm{Mn}: \mathrm{GaN}$.

For "pair" $\mathrm{Mn}_{\mathrm{Ga}}$ doping, we considered seven possible configurations in the 72-atom cell, with the two substitutional $\mathrm{Mn}$ atoms at various distances (ranging from $3.18 \AA$ to $6.10 \AA$ ) and in different spin states. In each geometry, one $\mathrm{Mn}$ atom $\left(\mathrm{Mn}_{\mathrm{I}}\right)$ is located at the origin, while the coordinates of the other $\mathrm{Mn}$ atom $\left(\mathrm{Mn}_{\mathrm{II}}\right)$ are given in terms of the primitive lattice vectors in the wurtzite structure. To reduce the effect of $\mathrm{Mn}-\mathrm{Mn}$ interactions between adjacent 
TABLE II. The Mn-Mn distances before $\left(d_{0}\right)$ and after $(d)$ relaxation, the relative total energy of the ground state $E_{\mathrm{FM}}$, and the total energy differences $\Delta E_{\mathrm{FM}-\mathrm{AFM}}$ of the ferromagnetic coupling relative to the antiferromagnetic coupling for the various $\mathrm{Mn}_{\mathrm{II}}$ positions for the pair $\mathrm{Mn}$ in wurtzite $\mathrm{GaN}$, with $\mathrm{Mn}_{\mathrm{I}}$ located at the origin and the coordinates of $\mathrm{Mn}_{\mathrm{II}}$ are given in terms of the primitive lattice vectors in the wurtzite structure.

\begin{tabular}{lcccc}
\hline \hline Coordinates of $\mathrm{Mn}_{\mathrm{II}}$ & $\begin{array}{c}d_{0} \\
(\AA)\end{array}$ & $\begin{array}{c}d \\
(\AA)\end{array}$ & $\begin{array}{c}E_{\mathrm{FM}} \\
(\mathrm{meV} / \mathrm{cell})\end{array}$ & $\begin{array}{c}\Delta E_{\mathrm{FM}-\mathrm{AFM}} \\
(\mathrm{meV} / \mathrm{Mn})\end{array}$ \\
\hline$(1 / 3,2 / 3,1 / 2)$ & 3.180 & 3.083 & 82.6 & -92.4 \\
$(1,0,0)$ & 3.189 & 3.091 & 0 & -130.1 \\
$(4 / 3,2 / 3,1 / 2)$ & 4.515 & 4.436 & 312.3 & -10.3 \\
$(0,0,1)$ & 5.186 & 5.116 & 402.9 & -24.5 \\
$(1 / 3,5 / 3,1 / 2)$ & 5.524 & 5.469 & 350.2 & -4.9 \\
$(4 / 3,8 / 3,0)$ & 5.532 & 5.471 & 322.3 & -29.8 \\
$(1,1,1)$ & 6.088 & 6.014 & 358.4 & -52.4 \\
\hline \hline
\end{tabular}

cells, a larger 108-atom cell is used for the $\mathrm{Mn}_{\mathrm{II}}$ atoms located at $(1,1,1)$ and $(1,2,1)$. For all structures, the nonmagnetic state is much higher in energy than FM or AFM. The distance between $\mathrm{Mn}$ atoms and the energy difference for the FM and AFM states for all configurations in the 72atom cell are compiled in Table II. For all the pair-Mn-doped structures considered, FM coupling is found to be the most favorable, regardless of the distance and orientation, which is in agreement with Refs. 45 and 48. The most favorable configuration is such that the nearest-neighboring $\mathrm{Mn}$ atoms substitute the in-plane Ga atoms [see Fig. 1(a)] with a mean moment of $3.82 \mu_{B} / \mathrm{Mn}$. The next most favorable structure is where the two Mn atoms substitute nearest-neighbor out-ofplane Ga sites [see Fig. 1(b)], with a mean moment of 3.79 $\mu_{B} / \mathrm{Mn}$. For these two structures, we also tried 32- and 96atom cells and confirmed that FM is more stable in both cases.

Considering the fact that the nearest $\mathrm{Mn}-\mathrm{Mn}$ distance in bulk $\alpha$-Mn is $2.25-2.94 \AA$, which is between the Ga-N $(1.95 \AA)$ and the Ga-Ga $(3.19 \AA)$ distances in pure GaN, we also investigated the possibility of $\mathrm{Mn}-\mathrm{Mn}$ bonded clustering (i.e., with no $\mathrm{N}$ atoms in between the two $\mathrm{Mn}$ atoms). Four structures are considered in the 72-atom cell, as depicted in Fig. 2. Both structures I and II contain an adjacent $\mathrm{Mn}_{\mathrm{Ga}}$ $+\mathrm{Mn}_{\mathrm{N}}$ complex, where the $\mathrm{Mn}$ atom pair is parallel and perpendicular to the (0001) plane, respectively. Structures III and IV involve two neighboring $\mathrm{Mn}_{\mathrm{Ga}}$ atoms with a $\mathrm{N}$ vacancy in between. The Mn-Mn pairs tend to cluster by contracting the distances between them for structures I, III, and IV. Interestingly, for structure II, the Mn-Mn pair shifts away from the host plane and the Mn-Mn distance is elongated by $12.8 \%$. The formation energy and magnetic moments for the various magnetic states are listed in Table I. The most favorable configuration is structure IV with an AFM ground state. But overall, the formation energies are much higher than the substitutional pair $\mathrm{Mn}_{\mathrm{Ga}}$, and thus we conclude that these Mn-Mn bonded configurations are unfavorable and will not play an important role in $\mathrm{Mn}: \mathrm{GaN}$.

Turning now to the consideration of polydoping, on substituting three $\mathrm{Mn}$ atoms on cation sites, we find that they
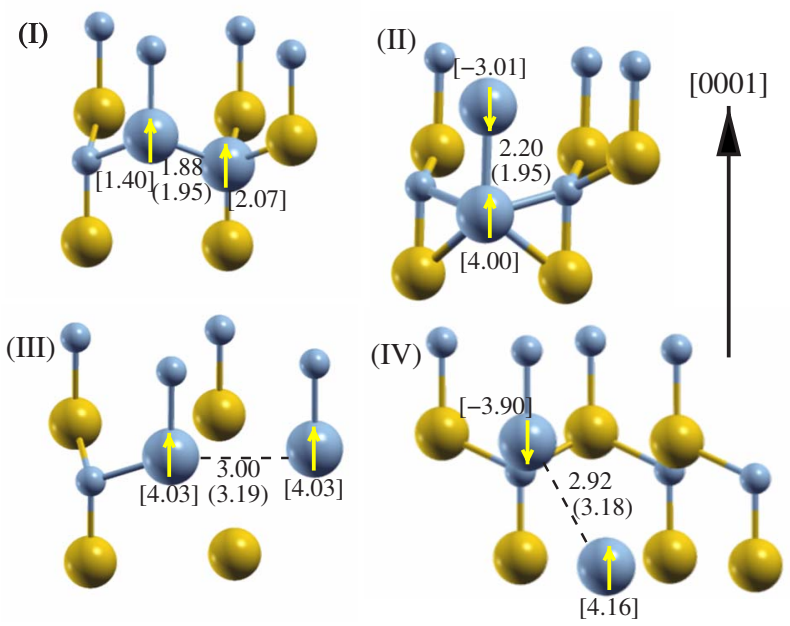

FIG. 2. (Color online) Configurations involving direct Mn-Mn interactions, where the large gray (blue) circles with arrows represent $\mathrm{Mn}$, the large gray (yellow) circles Ga atoms, and the small circles $\mathrm{N}$ atoms. Other notations are the same as in Fig. 1.

prefer to be close together. The energetically favored structures are the ones obtained by adding an extra Mn next to the favorable pair configurations. Significantly, the presence of the third Mn atom fundamentally changes the magnetic coupling, as expected by Kang and Chang. ${ }^{82}$ For the most favorable configurations of 3- $\mathrm{Mn}_{\mathrm{Ga}}$-doping systems [Fig. 1(c)], where the $\mathrm{Mn}$ atoms occupy nearest-neighboring Ga sites parallel to the (0001) plane, 72-atom cell calculations predict that the FM state with a net spin of $3.78 \mu_{B} / \mathrm{Mn}$ is more favorable than the AFM state with a net spin of $1.24 \mu_{B} / \mathrm{Mn}$, by $0.41 \mathrm{eV}$ per cell. However, both the 96- and 128-atom cell calculations predict that the AFM state is more favorable by 55 and $68 \mathrm{meV}$ than the FM state, respectively, and give a converged net moment of $0.91 \mu_{B} / \mathrm{Mn}$. For the next most favorable structure [Fig. 1(c)], all of the 72-, 96-, and 108atom cell calculations show that the AFM state is more favorable than the corresponding FM states, by 8, 44, and $52 \mathrm{meV}$ respectively, with a net spin moment of 0.92 $\mu_{B} / \mathrm{Mn}$. We should point out here that for the $3-\mathrm{Mn}_{\mathrm{Ga}}$-doping systems, the coupling strongly depends on the distribution of the dopants. For example, for a zigzag in-plane structure (a less favorable configuration), the FM state with a net spin moment of $3.8 \mu_{B} / \mathrm{Mn}$ is found to be more stable than all the possible AFM alignments, as calculated in the 72- and 128atom supercells.

For $4-\mathrm{Mn}_{\mathrm{Ga}}$ doping, we place the fourth $\mathrm{Mn}$ atom adjacent to the favorable $3-\mathrm{Mn}_{\mathrm{Ga}}$ structures, resulting in the configurations shown in Figs. 1(e) and 1(f), where the former is the most stable. We also tried several configurations where the fourth $\mathrm{Mn}$ is far from the $3-\mathrm{Mn}_{\mathrm{Ga}}$ structures and confirmed that such structures are clearly energetically unfavorable. For both stable structures, states containing AFM coupling are still favored. Moreover, the energy difference between the FM and AFM states increases significantly and reaches $0.81 \mathrm{eV}$ (per 96-atom cell) and $0.89 \mathrm{eV}$ (per 108atom cell) for the most favorable $4-\mathrm{Mn}_{\mathrm{Ga}}$ pyramid structure [Fig. 1(e)], where the out-of-plane Mn atom couples antiparallel to those of the three in-plane $\mathrm{Mn}$ atoms, resulting in a 
net spin moment of $1.43 \mu_{B} / \mathrm{Mn}$. For the next most favorable configuration of $4 \mathrm{Mn}_{\mathrm{Ga}}$, which corresponds to an in-plane rhombus structure [Fig. 1(f)], again 128-atom cell calculations predict that the AFM state with a net spin of 0.04 $\mu_{B} / \mathrm{Mn}$ is more favorable by $110 \mathrm{meV}$ per supercell as compared to the FM state.

For the $5-\mathrm{Mn}_{\mathrm{Ga}}$ bipyramid structure [Fig. 1(g)] with a net spin of $0.97 \mu_{B} / \mathrm{Mn}, 108$-atom cell calculations predict that the FM state is not stable, i.e., the self-consistent field calculation converges the initial FM configuration to an AFM solution. Strikingly, 256-atom cell calculations show that the AFM state is more stable than the FM state by $156 \mathrm{meV}$ per cell; i.e., for the larger supercell, the FM state could be stabilized, but is still energetically less favorable. These results clearly demonstrate that as the size of the Mn clusters increases, AFM coupling is favorable. This behavior is in contrast to that of purely substitutional Mn:GaAs clusters, where the FM alignment is predicted to be energetically favorable using projector augmented-wave calculations. ${ }^{84}$ However, Mahadevan and Zunger predicted that in Mn:GaAs, the complex $\mathrm{Mn}_{\mathrm{Ga}}-\mathrm{Mn}_{i}-\mathrm{Mn}_{\mathrm{Ga}}$ is actually more stable than pure $\mathrm{Mn}_{\mathrm{Ga}}$ clusters. ${ }^{85}$ Furthermore, our results are in contradiction with those by Sandratskii et al. ${ }^{57}$ who predicted that the ground state of Mn clusters in Mn:GaN (up to $4 \mathrm{Mn}$ atoms in the 64-atom cell) is FM, with the Mn atomic moment depending weakly on both $\mathrm{Mn}$ concentration and clustering, varying from $3.37 \mu_{B}$ to $3.43 \mu_{B}$. We attribute this discrepancy to (i) the smaller supercell they used, i.e., a 64-atom $(2 a \times 2 a$ $\times 2 a$ ) zinc blende structure supercell was used throughout for doping up to $4 \mathrm{Mn}$ atoms and (ii) no atomic relaxation was taken into account. ${ }^{57}$ The former factor is understandable since we show that smaller supercells do tend to favor FM coupling due to interactions between neighbouring supercells, as demonstrated above and also found for $\mathrm{Cr}: \mathrm{GaN}{ }^{49,50}$ To address the importance of the role of atomic relaxation, we performed further calculations for the structures shown in Figs. 1(c) and 1(e) using the unrelaxed ideal $\mathrm{GaN}$ host structure. Indeed, for the $3-\mathrm{Mn}_{\mathrm{Ga}}$ cluster shown in Fig. 1(c), both 96- and 128-atom cells actually predict that the FM state is more stable than the AFM state by 21 and $14 \mathrm{meV}$, respectively. However, for the $4-\mathrm{Mn}_{\mathrm{Ga}}$ configuration shown in Fig. 1(e), both 96- and 108-atom cells predict that for the unrelaxed host structure, AFM state is more stable due to the stronger magnetic coupling therein. Thus, careful consideration of the effect of supercell size and atomic relaxation is crucial in determining the magnetic coupling for DMS systems.

We also considered the possibility of substitutionalinterstitial complexes. Since single interstitial doping of $\mathrm{Mn}$ in $\mathrm{GaN}$ is highly unfavorable, we consider various substitutional-interstitial complexes containing only one $\mathrm{Mn}_{i-O}$ [Figs. 1(h)-1(k)]. Structures containing AFM coupling are again found to be more favorable than FM states by $51 \mathrm{meV}$ for the structure shown in Fig. 1(h) and $98 \mathrm{meV}$ for the structure shown in Fig. 1(i), as calculated in a 72-atom cell. For the configurations shown in Figs. 1(j) and 1(k), FM states are not stable in the 96-atom cell. Again, the inclusion of the extra interstitial $\mathrm{Mn}$ atom dramatically changes the magnetic coupling. For example, adding one $\mathrm{Mn}_{i-O}$ at the center of the configuration shown in Fig. 1(e) leads to the structure shown in Fig. 1(k), where the four surrounding $\mathrm{Mn}_{\mathrm{Ga}}$ atoms now couple ferromagnetically. Furthermore, in all the complexes containing more than $2 \mathrm{Mn}_{\mathrm{Ga}}$, there is a $\mathrm{FM}$ alignment between the $\mathrm{Mn}_{\mathrm{Ga}}$ atoms, and the coupling between $\mathrm{Mn}_{i-O}$ and $\mathrm{Mn}_{\mathrm{Ga}}$ atoms is always antiparallel. Note that in the complexes, the $\mathrm{Mn}_{i-O}$ and $\mathrm{Mn}_{\mathrm{Ga}}$ atoms are directly bonded, while in the pure $\mathrm{Mn}_{\mathrm{Ga}}$ clusters, $\mathrm{Mn}$ atoms are bonded via $\mathrm{N}$ atoms. Popovic et al. ${ }^{40}$ investigated the substitutional $\mathrm{Mn}_{\mathrm{Ga}}$ and interstitial single-doping $\mathrm{Mn}_{i-O}$ in $\mathrm{GaN}$ and suggested the formation of Coulomb stabilized complexes such as $\mathrm{Mn}_{\mathrm{Ga}} \mathrm{Mn}_{i-O} \mathrm{Mn}_{\mathrm{Ga}}$, where the coupling between $\mathrm{Mn}_{i-O}$ and two $\mathrm{Mn}_{\mathrm{Ga}}$ is antiferromagnetically aligned. Our DFT calculations confirm such coupling [see Figs. 1(i) and 1(h)]. However, the calculated formation energies of such complexes are significantly higher than the same concentration of the purely substitutional $\mathrm{Mn}_{\mathrm{Ga}}$ clusters, indicating that such $\mathrm{Mn}_{\mathrm{Ga}} \mathrm{Mn}_{i-O} \mathrm{Mn}_{\mathrm{Ga}}$ complexes may not play a significant role in Mn:GaN. From Table I, overall, it can be clearly seen that the formation energies for the substitutionalinterstitial complexes are much higher than the same concentration pure substitutional configurations, showing that the directly Mn-Mn bonded defects are rather unlikely, which is in agreement with recent experiments. ${ }^{86,87}$

Our results clearly demonstrate that the stability and magnetic properties of small Mn clusters can be fundamentally altered by the presence of a third and more Mn atoms. This is highlighted in Fig. 3, where we display the spin density contour plots of the magnetic ground states for the single, pair [Fig. 1(a)], and trio [Fig. 1(c)] structures in the (0001) plane obtained from the cubic 96-atom supercell calculations. The preference for AFM over FM for larger Mn clusters in Mn:GaN might actually not be so surprising when one considers that the bulk $\alpha$-Mn (Ref. 72) phase is AFM, and further for the gas phase $\mathrm{Mn}_{n}$ clusters, while for smaller sizes $(n \leqslant 6)$ FM and AFM are competitive, for larger ones $(n \geqslant 7)$, AFM is clearly favored. ${ }^{88}$

\section{B. Charged states}

So far, we have demonstrated clustering behavior in Mn: $\mathrm{GaN}$, where the valence state of the $\mathrm{Mn}$ ion is assumed to be neutral, i.e., $\mathrm{Mn}^{3+}\left(d^{4}\right)$. However, there is experimental evidence showing that the oxidation states are important in $\mathrm{Mn}$ :GaN samples, where typically $\operatorname{Mn}^{2+}\left(d^{5}\right)$ and $\operatorname{Mn}^{3+}\left(d^{4}\right)$ coexist. $^{21,52,53,62,63}$ Considering the large number of possible configurations and states involved, we report calculations on selected structures based on our neutral state results, namely, purely substitutional $\mathrm{Mn}_{\mathrm{Ga}}$ clusters.

We first investigate whether the clustering behavior is still preferred for the charged states. To do this, we place two $\mathrm{Mn}_{\mathrm{Ga}}$ atoms in the 72-atom cell with the $\mathrm{Mn}$ atoms in two structures; one is a "near" configuration [see Fig. 1(a)] and the other is a "far" configuration (with a Mn-Mn distance of $6.088 \AA$ ). Both with and without spin polarization, the calculations predict that clustering behavior is preferred for all the charged states considered (from 1 to -2 ). The relative energies between the nonmagnetic, FM, and AFM states and the atomic magnetic moments are listed in Table III. Similarly, for the doping of three $\mathrm{Mn}_{\mathrm{Ga}}$ atoms, we calculate the 

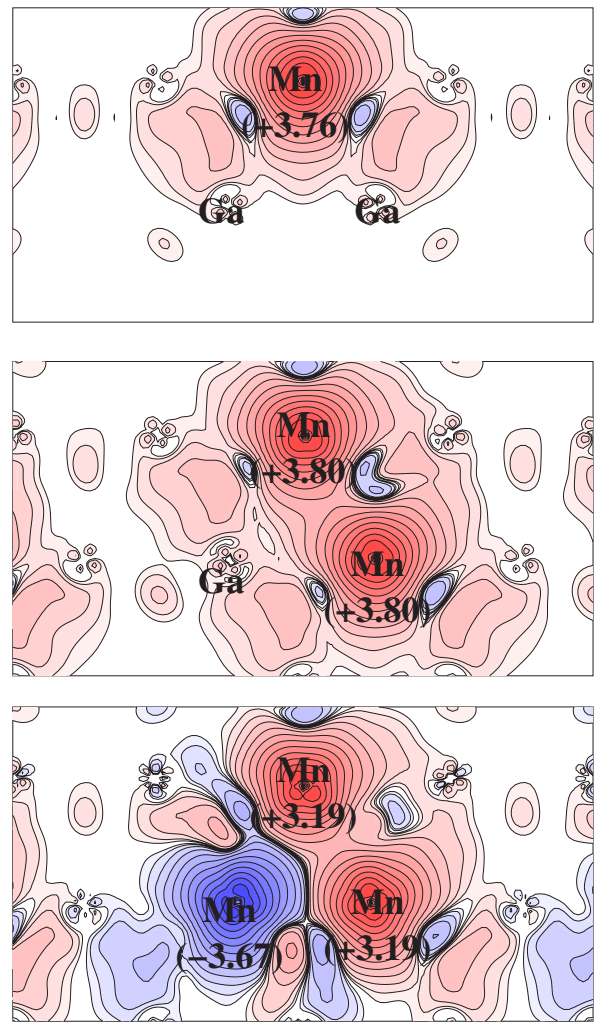

FIG. 3. (Color online) Spin density contour plots for the ground magnetic states of single (upper), pair (middle) [see Fig. 1(a)], and trio (lower) [see Fig. 1(c)] configurations in the (0001) plane as calculated in the cublic 96-atom cell. The values of the atomic magnetic moments are indicated.

energy difference between the nonmagnetic states for the near and far configurations with different charged states. Again, clustering is predicted for the charged states considered (from +2 to -3 ). Thus, we conclude that despite repulsive Coulomb forces between the isocharged ions, clustering is still preferred. Similar behavior was predicted for $\mathrm{Mn}: \mathrm{GaAs}$ systems. ${ }^{42}$

Now we study the magnetic coupling for the neutral and the charged state $\mathrm{Mn}_{\mathrm{Ga}}$ clusters. The energetics and magnetic moments for singlet, pair, trio, and quarto pure $\mathrm{Mn}_{\mathrm{Ga}}$ clusters, as calculated in the cubic 96-atom cell, are summarized in Table IV. Figure 4 summarizes the formation energies as a function of the Fermi energy for various configurations under $\mathrm{N}$-rich conditions. The slopes of the formation energies characterize the charged states, and the transition from one charged state to another is shown by the crossover of the energetically lowest lines.

First, we consider the single $\mathrm{Mn}_{\mathrm{Ga}}$ atom in $\mathrm{GaN}$. In the tetrahedral crystal-field environment, the neutral $\mathrm{Mn}^{3+}\left(d^{4}\right)$ ion has an $e^{2} t_{2}^{2}$ configuration (with a hole for one $t_{2}$ orbital) for the high spin state $\left(4 \mu_{B} /\right.$ cell $)$ and an $e^{4} t_{2}^{0}$ configuration for the low spin state $\left(0 \mu_{B} /\right.$ cell $) .{ }^{80}$ The presence of the negatively charged $\mathrm{Mn}^{2+}\left(d^{5}\right)$ can be well understood because it leads to an energetically favorable half-filled $e^{2} t_{2}^{3}$ configuration with a moment of $5 \mu_{B} /$ cell. Another favorable state is formed by removing one electron from the ion, leading to an $e^{2} t_{2}^{1}$ configuration, with a moment of $3 \mu_{B}$ /cell. The unfavorable +2 state indicates that further removal of an electron is not easy. Our results predict that the single $\mathrm{Mn}_{\mathrm{Ga}}$ in $\mathrm{GaN}$ can be stabilized in various valence states with diverse magnetic moments depending on the Fermi level, i.e., $n$ type or $p$ type of the host semiconductor, as shown in Fig. 4(a).

For pair doping and polydoping, we study the structures shown in Figs. 1(a), 1(c), and 1(e) in various charged states. Importantly, for the pair-doping configuration, Fig. 1(a), while the neutral and -1 charged states (adding one electron) predict that FM couping is more stable than AFM couping, calculations for the -2 charged state and more negatively charged states predict that AFM coupling is more stable. We have confirmed this interesting behavior for both 32- and 96-atom supercells. This is in agreement with the model and band structure calculations by Dalpian et al., ${ }^{81,83}$ in which they attributed this behavior to the competition between the crystal-field splitting and the exchange splitting. However, we argue that the AFM -2 state might not be responsible for the low-mean-magnetic-moment value since this state and further negatively charged states are energetically unfavorable, as shown in Fig. 4(b). The "doped electrons" in the FM state are equally shared by the two Mn atoms and the atomic magnetic moment of the Mn ions in the pair-doping -2 state

TABLE III. Relative total energies (in eV) and atomic magnetic moments (in parentheses and in $\mu_{B} / \mathrm{Mn}$ ) for the nonmagnetic, FM, and AFM states for the "near-pair" and "far-pair" $\mathrm{Mn}_{\mathrm{Ga}}$ atoms in various charged states, as calculated in the 72-atom supercell. The quantities in boldface type are the ground states.

\begin{tabular}{|c|c|c|c|c|c|c|}
\hline \multirow[b]{2}{*}{ Charged states } & \multicolumn{3}{|c|}{ Near-pair } & \multicolumn{3}{|c|}{ Far-pair } \\
\hline & Nonmagnetic & FM & AFM & Nonmagnetic & FM & AFM \\
\hline 1 & 1.340 & $\begin{array}{c}0 \\
(3.39,3.37)\end{array}$ & $\begin{array}{c}0.238 \\
(2.66,-3.50)\end{array}$ & 1.623 & $\begin{array}{c}0.332 \\
(3.39,3.39)\end{array}$ & $\begin{array}{c}0.443 \\
(3.36,-3.37)\end{array}$ \\
\hline 0 & 1.952 & $\begin{array}{c}0 \\
(3.80,3.80)\end{array}$ & $\begin{array}{c}0.237 \\
(3.53,-3.64)\end{array}$ & 2.444 & $\begin{array}{c}0.347 \\
(3.81,3.81)\end{array}$ & $\begin{array}{c}0.444 \\
(3.78,-3.79)\end{array}$ \\
\hline-1 & 2.778 & $\begin{array}{c}0 \\
(4.20,4.20)\end{array}$ & $\begin{array}{c}0.113 \\
(4.00,-4.03)\end{array}$ & 3.441 & $\begin{array}{c}0.227 \\
(4.19,4.19)\end{array}$ & $\begin{array}{c}0.299 \\
(4.18,-4.18)\end{array}$ \\
\hline-2 & 3.645 & $\begin{array}{c}0.309 \\
(4.55,4.55)\end{array}$ & $\begin{array}{c}0 \\
(4.41,-4.41)\end{array}$ & 4.440 & $\begin{array}{c}0.075 \\
(4.57,4.57)\end{array}$ & $\begin{array}{c}0.114 \\
(4.56,-4.56)\end{array}$ \\
\hline
\end{tabular}


TABLE IV. Atomic magnetic moments (M.M.) and the energy difference between the FM and AFM phases for the various configurations with different valence states, calculated in the 96-atom cell.

\begin{tabular}{|c|c|c|c|}
\hline $\begin{array}{l}\text { Valence } \\
\text { states }\end{array}$ & $\begin{array}{l}\text { M.M. on Mn } \\
\text { in FM state }\end{array}$ & $\begin{array}{l}E_{\mathrm{FM}-\mathrm{AFM}} \\
(\mathrm{eV} / \text { cell })\end{array}$ & $\begin{array}{l}\text { M.M. on Mn } \\
\text { in AFM state }\end{array}$ \\
\hline \multicolumn{4}{|c|}{ Single $\mathrm{Mn}_{\mathrm{Ga}}$ configuration } \\
\hline+2 & 1.89 & & \\
\hline+1 & 2.91 & & \\
\hline 0 & 3.79 & & \\
\hline-1 & 4.58 & & \\
\hline \multicolumn{4}{|c|}{ Pair $\mathrm{Mn}_{\mathrm{Ga}}$; Fig. 1(a) configuration } \\
\hline+4 & $1.96 ; 1.96$ & -0.087 & $1.84 ;-1.84$ \\
\hline+2 & $2.94 ; 2.93$ & -0.102 & $2.46 ;-2.49$ \\
\hline+1 & $3.39 ; 3.37$ & -0.211 & $2.66 ;-3.50$ \\
\hline 0 & $3.80 ; 3.80$ & -0.202 & $3.53 ;-3.64$ \\
\hline-1 & $4.20 ; 4.20$ & -0.0570 & $4.00 ;-4.03$ \\
\hline-2 & $4.55 ; 4.55$ & 0.339 & $4.41 ;-4.41$ \\
\hline \multicolumn{4}{|c|}{ Trio $\mathrm{Mn}_{\mathrm{Ga}}$; Fig. 1(c) configuration } \\
\hline+6 & $1.97 ; 2.13 ; 2.13$ & -0.0936 & $2.13 ;-2.01 ;-2.01$ \\
\hline+2 & $3.25 ; 3.30 ; 3.30$ & 0.0462 & $3.73 ;-2.36 ;-2.36$ \\
\hline+1 & $3.58 ; 3.55 ; 3.55$ & 0.0302 & $3.69 ;-2.86 ;-2.86$ \\
\hline 0 & $3.86 ; 3.80 ; 3.80$ & 0.0774 & $3.84 ;-3.24 ;-3.24$ \\
\hline-1 & $4.11 ; 4.05 ; 4.05$ & 0.213 & $3.94 ;-3.62 ;-3.62$ \\
\hline-2 & $4.38 ; 4.31 ; 4.31$ & 0.600 & $4.11 ;-3.94 ;-3.94$ \\
\hline-3 & $4.55 ; 4.52 ; 4.52$ & 0.723 & $4.12 ;-4.27 ;-4.27$ \\
\hline \multicolumn{4}{|c|}{ Quarto $\mathrm{Mn}_{\mathrm{Ga}}$; Fig. 1(e) configuration } \\
\hline+8 & $2.06 ; 2.05 ; 2.18 ; 2.18$ & -0.186 & $-1.59 ; 1.85 ; 1.96 ; 1.96$ \\
\hline+2 & & & $-1.69 ; 3.00 ; 3.12 ; 3.13$ \\
\hline+1 & & & $-2.75 ; 3.04 ; 3.13 ; 3.13$ \\
\hline 0 & $3.13 ; 3.31 ; 3.42 ; 3.42$ & 0.790 & $-3.52 ; 3.13 ; 3.25 ; 3.25$ \\
\hline-1 & $3.48 ; 3.48 ; 3.57 ; 3.57$ & 0.782 & $-3.76 ; 3.45 ; 3.51 ; 3.51$ \\
\hline-2 & $3.64 ; 3.63 ; 3.68 ; 3.68$ & 0.798 & $-3.90 ; 3.81 ; 3.82 ; 3.82$ \\
\hline-3 & $3.51 ; 2.99 ; 3.06 ; 3.06$ & 0.512 & $-3.80 ; 4.05 ; 4.01 ; 4.01$ \\
\hline-4 & $3.42 ; 3.25 ; 3.27 ; 3.27$ & 0.296 & $-3.64 ; 4.24 ; 4.19 ; 4.19$ \\
\hline
\end{tabular}

is very close to the value of the single $\mathrm{Mn}^{2+}\left(d^{5}\right)$ ion. Similar behavior is also found in large cluster configurations. For trio doping of $\mathrm{Mn}_{\mathrm{Ga}}$ [see structure in Fig. 1(c)], for all the energetically favorable valence states $(+2,0,-1$, and -2$)$ shown in Fig. 3(c), the AFM state is more stable than the FM state. For the experimentally observed oxidation (negatively charged) states, the energy difference between AFM and FM is significantly enhanced compared to that of the neutral state. For quarto doping of $\mathrm{Mn}_{\mathrm{Ga}}$ [the structure in Fig. 1(e)], where the positively charged +1 and +2 states are found to be unstable in the FM state, the initial FM configuration converged into AFM solutions. For the very highly positive +8 state, the FM state has an energy gain of $46.5 \mathrm{meV} / \mathrm{Mn}$ over the AFM state as calculated in the 96-atom cell. For all the favorable neutral and charged states $(+1,0,-1$, and -2$)$ shown in Fig. 3(d), the AFM state is always favored over the FM state.
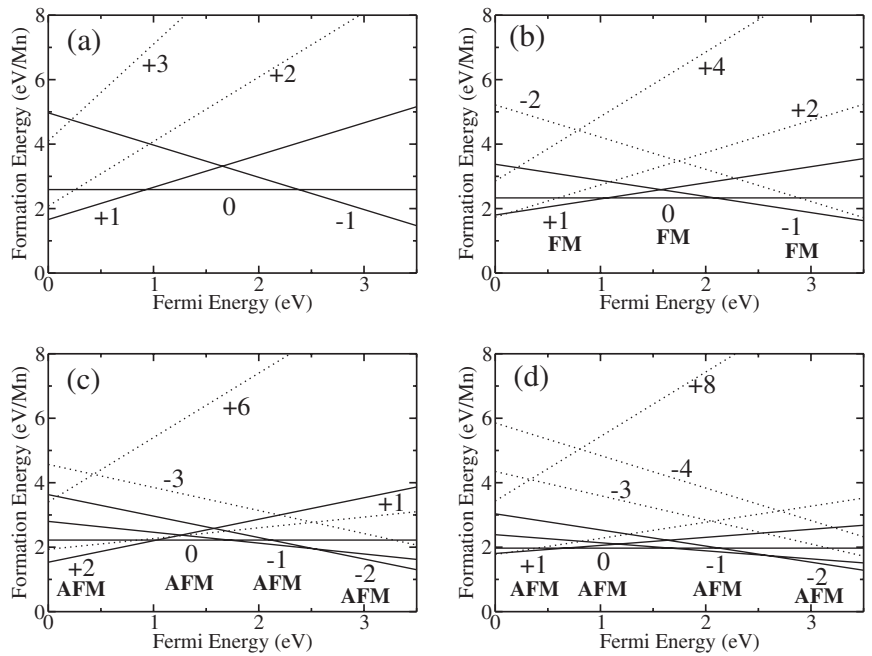

FIG. 4. Formation energy (under N-rich conditions) for the neutral and charged states for various polydoping $\mathrm{Mn}_{\mathrm{Ga}}$ configurations as calculated in the 96-atom cell. (a) Single $\mathrm{Mn}_{\mathrm{Ga}}$, (b) the pair $\mathrm{Mn}_{\mathrm{Ga}}$ structure shown in Figs. 1 (a) and 1 (c) the trio $\mathrm{Mn}_{\mathrm{Ga}}$ structure shown in Figs. 1(c) and 1(d) the quarto $\mathrm{Mn}_{\mathrm{Ga}}$ structure shown in Fig. 1(e). Dotted lines indicate unstable charged states. The Fermi energy, $E_{F}=0$, corresponds to the top of the valence band of pure GaN. The magnetic ground states (FM versus AFM) are also in (b), (c), and (d). In (a), the values of the magnetic moment per cell are $3 \mu_{B}, 4$ $\mu_{B}$, and $5 \mu_{B}$ for the +1 , neutral, and -1 charged states, respectively. Under Ga-rich conditions, the atomic chemical potential of gallium is reduced by $1.04 \mathrm{eV}$ (the heat of formation of $\mathrm{GaN}$ ) and the manganese atomic potential is reduced by $0.46 \mathrm{eV}$ (as related to the use of bulk GaMn versus $\mathrm{MnN}$ ). This results in the formation energies being shifted upward by $0.58 \mathrm{eV}$.

To summarize our charged states results, our firstprinciples calculations demonstrate that for a given $\mathrm{Mn}_{\mathrm{Ga}}$ cluster configuration, various (neutral and both positively and negatively charged) states can, in principle, be stabilized. Interestingly, experimental results so far only reported the coexistence of the neutral and negatively charged states, with the latter states being found dominant in some cases. ${ }^{61-63}$ Presumably, this can be understood by the well-known fact that as-grown $\mathrm{GaN}$ is usually $n$ type (with a surplus of electrons), and $p$-type conductivity is difficult to obtain..$^{89,90}$ Moreover, our results clearly show that the experimentally observed oxidation states in Mn:GaN favor the AFM state for the pair and polyclustering configurations. Again, this indicates that the "giant cluster moment" in $\mathrm{Mn}: \mathrm{GaN}$ is not applicable for "larger" $(>2)$ Mn-cluster-doped GaN.

\section{DISCUSSION}

Importantly, but not surprisingly, we find that the electronic character of Mn:GaN depends sensitively on the valence states. We display the density of states (DOS) for the neutral and charged states of single-, pair- [Fig. 1(a)], and poly-Mn $\mathrm{Ga}_{\text {a }}$ [Figs. 1(c) and 1(e)] configurations in Figs. 5-7, respectively, at different concentrations by using 32- and 96atom cells. It is found that the band gap of the host semiconductor serves as the "arena" of the Mn- $3 d$-band electrons. 

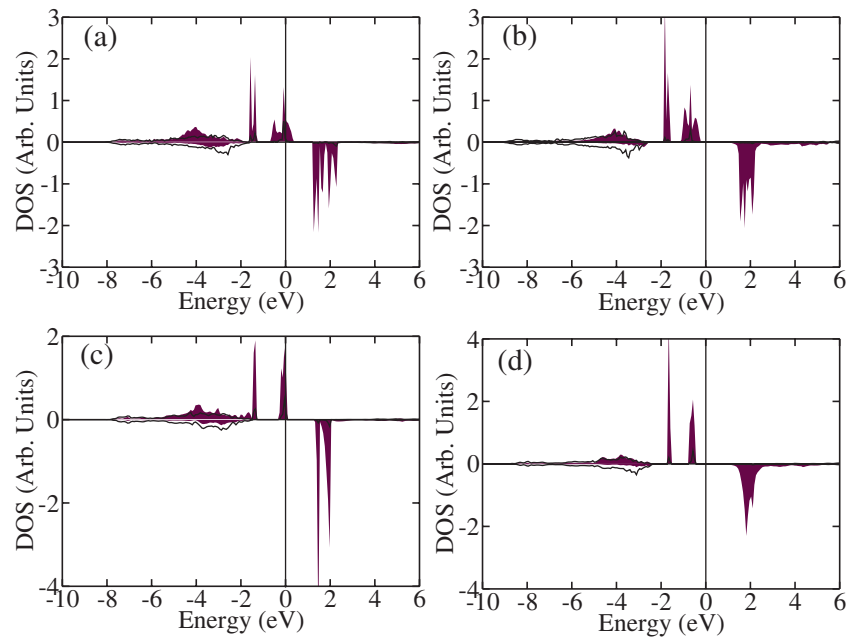

FIG. 5. (Color online) Projected DOS for single $\mathrm{Mn}_{\mathrm{Ga}}$ in the [(a) and (c)] neutral and [(b) and (d)] -1 charged states with different supercells [(a) and (b)] using a 32-atom cell and [(c) and (d)] using a 96-atom cell. The filled gray areas are for Mn $3 d$ states and the solid lines are for the singly Mn-bonded $\mathrm{N}$ atom $2 p$ states.

For neutral single and pair $\mathrm{Mn}_{\mathrm{Ga}}$, half-metallicity is predicted at both concentrations, which is in agreement with previous theoretical studies. ${ }^{35,39,41,44,47,59}$ However, for the negatively charged state $\left(-1\right.$ for single $\mathrm{Mn}_{\mathrm{Ga}}$ and the -2 charged state for pair $\mathrm{Mn}_{\mathrm{Ga}}$ ), it is clearly insulating. This is because the doped electron(s) will fill the holes and move the Fermi level upward and also gives the larger atomic moments. For larger clusters [Figs. 1(c) and 1(e)], both neutral and negatively charged state calculations show highly insulating character with numerous states situated in the band gap. Note that experimental results show that the Mn:GaN systems are highly resistive, ${ }^{22}$ and even semi-insulating ${ }^{60}$ or insulating. ${ }^{19,24}$ Thus, our first-principles results indicate that the embedded clusters, particularly their negatively charged
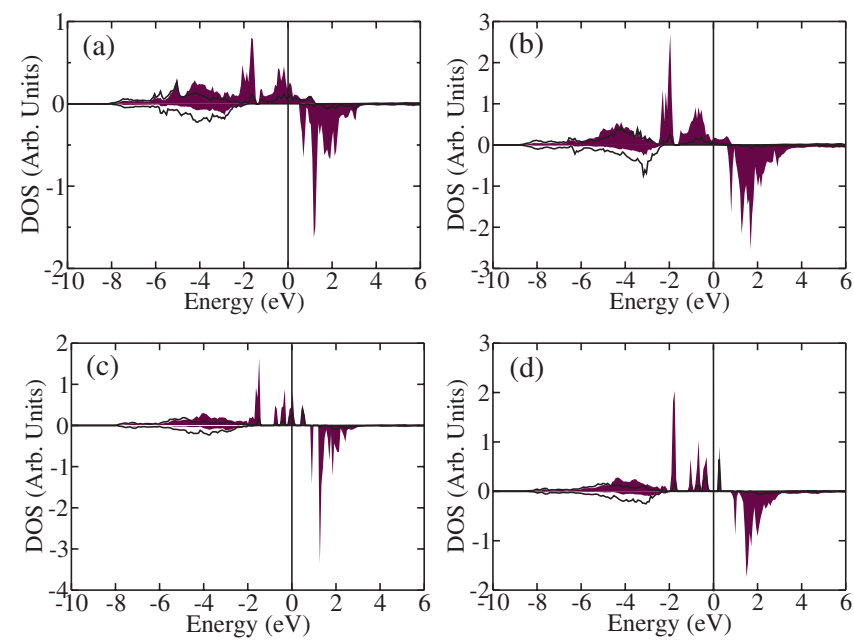

FIG. 6. (Color online) Projected DOS for pair-doping $\mathrm{Mn}_{\mathrm{Ga}}$ in the [(a) and (c)] neutral and [(b) and (d)] -1 charged states with different supercells [(a) and (b)] using a 32-atom cell and [(c) and (d)] using a 96-atom cell. The filled gray areas are for Mn $3 d$ states and the solid lines are for the singly Mn-bonded $\mathrm{N}$ atom $2 p$ states.
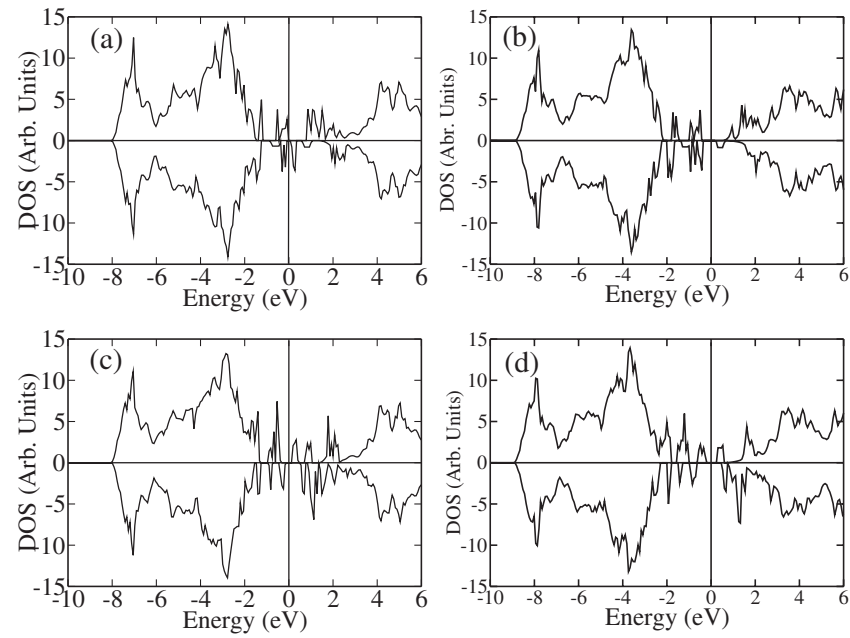

FIG. 7. Total DOS for polydoping $\mathrm{Mn}_{\mathrm{Ga}}$ in the [(a) and (c)] neutral and [(b) and (d)] negatively charged states using the cubic 96-atom supercell: (a) neutral and (b) -2 charged state of the structure shown in Fig. 1(c) $\left(3 \mathrm{Mn}_{\mathrm{Ga}}\right)$ and (c) neutral and (d) -2 charged state of the structure shown in Fig. $1(\mathrm{e})\left(4 \mathrm{Mn}_{\mathrm{Ga}}\right)$.

states, are responsible for the experimentally observed highly insulating properties in $\mathrm{Mn}: \mathrm{GaN}$.

Regarding the atomic structure, an important feature is that the clustering causes a strong local structural distortion for both neutral and charged states, with the Mn-Mn distances contracted by up to $11 \%$ and $9.8 \%$ [neutral and -4 charged state of Fig. 1(e)] and 17\% [neutral Fig. 1(g)] as compared to the Ga-Ga distance in the host lattice, indicating a strongly attractive interaction between the $\mathrm{Mn}$ atoms. This can be contrasted to the case of a single $\mathrm{Mn}_{\mathrm{Ga}}$, where the $\mathrm{Mn}-\mathrm{N}$ bond is longer than the ideal Ga-N bond by $1.65 \%$ due to the larger atomic radius of $\mathrm{Mn}(1.61 \AA)$ than that of Ga $(1.36 \AA)$. In the substitutional clusters, the contracted $\mathrm{Mn}-\mathrm{Mn}$ distances are due to the strong atomic attraction and magnetic coupling. Again, our results demonstrate that atomic relaxation plays an important role in polydoping investigation in DMS, which is neglected in Refs. 57 and 84.

Another interesting feature is that the Mn embedded clusters are found around one $\mathrm{N}$ atom, which is in agreement with that in Refs. 57 and 84 for substitutional Mn:GaAs. There is a strong local polarization on the central $\mathrm{N}$ atoms (bonded to Mn); for example, $0.09 \mu_{B}, 0.19 \mu_{B}$, and $0.21 \mu_{B}$ are found for the structures shown in Figs. 1(a), 1(c), and 1(e), respectively, compared to $0.015 \mu_{B}$ on $\mathrm{N}$ for the case of isolated $\mathrm{Mn}_{\mathrm{Ga}}\left(d^{4}\right)$ and $0.027 \mu_{B}$ for the negatively charged $\mathrm{Mn}_{\mathrm{Ga}}\left(d^{5}\right)$ ions. For other neighboring $\mathrm{N}$ atoms, there are still noticeable magnetic moments, typically ranging between 0.01 and $0.1 \mu_{B}$, mainly depending on the coordination number to $\mathrm{Mn}$ atoms. The large moments on the $\mathrm{N}$ atoms are still due to hybridization between the $\mathrm{N} 2 p$ and $\mathrm{Mn} 3 d$ states and we attribute the strong hybridization found in soft $\mathrm{x}$-ray emission and absorption spectroscopy ${ }^{87}$ to the $\mathrm{N} 2 p$ and Mn $3 d$ states in embedded clustering configurations, rather than the much weaker single isolated $\mathrm{Mn}_{\mathrm{Ga}}$-doping configuration.

Based on our extensive first-principles investigations of $\mathrm{Mn}$ in GaN, we propose that the spatial distribution of the 
Mn ions is not random or homogeneous, as widely assumed in the literature. The dopants prefer to get close to each other by forming $\mathrm{Mn}-\mathrm{N}-\mathrm{Mn}$ bonded embedded clusters in the host semiconductor, maintaining the long-ranged wurtzite lattice. Moreover, even with the widely used pair-model description, we find that while the neutral and -1 negatively charged Mn-Mn pair is FM, the -2 charged states, where each Mn atom is a $\mathrm{Mn}_{\mathrm{Ga}}-d^{5}$ ion, and furthermore negatively charged states are AFM. For larger than pair configurations, both neutral and negatively charged states favor AFM coupling. The occurrence of the AFM coupling in the embedded clusters may play an important role in DMS systems, particularly in high-concentration samples. Various configurations with diverse magnetic structures and valence states will presumably coexist, where the statistical distribution, and resulting magnetism, will depend sensitively on the details of the growth conditions. Interestingly, "random distributed clusters" were experimentally observed in Cr:GaN samples. ${ }^{91}$ Note that from a long-range-scale point of view, the coexistence of various local embedded clustering does not violate the "uniformlike" distribution observed using techniques such as secondary ion mass spectroscopy. ${ }^{92}$ We expect that the resultant local clustering inhomogeneous distribution can be detected by techniques such as energy-filtered imaging ${ }^{91}$ and analytical transmission electron microscopy ${ }^{93}$ as recently demonstrated in $\mathrm{Cr}: \mathrm{GaN}$. Moreover, recent experiments show that various local structural environments coexist for $\mathrm{Mn}: \mathrm{GaN}$ at a concentration of $4.5 \% .{ }^{63}$ Such a scenario suggests that there is some variability in the structural environment of the Mn dopant and consequently different magnetic properties may result from different local structures. These, in turn, are expected to vary with growth conditions, illustrating the critical importance of careful process control in the growth of this material.

With this picture, one can further understand some hitherto puzzling experimental findings: (i) The striking discrepancy between the experimentally observed much lower magnetic moment, typically less than $0.5 \mu_{B} / \mathrm{Mn}$, and the previously expected $4 \mu_{B} / \mathrm{Mn}\left(0.5 \mu_{B} / \mathrm{Mn}\right)$ for the neutral (-1 charged) single $\mathrm{Mn}_{\mathrm{Ga}}$ value, can be attributed to the AFM coupling within the embedded clusters. Another source contributing to the lower mean moment may be due to surface segregation, as observed in experiments ${ }^{52,94}$ and theoretically studied by Wang et al. ${ }^{48}$ who demonstrated that the neutral pair Mn are AFM on surfaces due to the contracted Mn-Mn distances. (ii) Saturation magnetic moments are found to decrease with increasing Mn doping concentration, ${ }^{95}$ as can be naturally understood from the Mn atoms increasingly experiencing an attractive interaction and forming AFM clusters which result in a lower net magnetization. (iii) X-ray absorption experiments observed that the ordering of the Mn atoms along the [0001] direction is more imperfect than in the direction parallel to the substrate. ${ }^{53}$ This interesting behavior can be understood by considering that during the MBE growth process, the samples are grown layer by layer in the [0001] direction, and consequently that the embedded clusters are much easier to form along the $c$ axis than along the $a$ axis due to the attractive interaction. Recently, Fukushima et al. ${ }^{96}$ modeled this behavior and showed that spinodal decomposition growth conditions lead to quasi-one-dimensional nanostructures for both $\mathrm{Cr}: \mathrm{ZnTe}$ and Mn:GaN. (iv) To date, there is a large discrepancy in the reported measurements and theoretically calculated $T_{C}$. Recently, based on a mean-field description, Hynninen et al. ${ }^{58}$ highlighted the complexity using the energy difference between the FM and AFM states of various cluster-cluster interactions, where within the clusters, the interaction was assumed to be FM and the calculations were limited to the neutral states. Our results suggest that in reality, such a quantity could be even more complicated. The coexistence of FM and AFM coupling in various configurations with different charged states along with their spatial distribution (the orientation and the cluster-cluster distance) may explain the abnormally large variations for the reported values.

\section{CONCLUSION}

In summary, our systematic study clearly shows that the $\mathrm{Mn}$ atoms prefer to occupy Ga sites and have strong a tendency to form embedded clusters. Substitutional configurations are more favorable than substitutional-interstitial complexes. For neutral pair doping, while the coupling is FM regardless of the distance and orientation of the Mn atoms, for the negatively ( -2 and higher) charged states, the AFM state is favored. For Mn clusters larger than 2 atoms, for all the favorable neutral and charged states, AFM states are energetically preferred over the FM states. Thus, we conclude that the "giant magnetic moments," as predicted previously, are actually not applicable in the Mn:GaN DMS systems. We propose a picture where various cluster configurations with different valence states coexist and the statistical distribution and the associated magnetism depend sensitively on the growth conditions. We also emphasize that the effect of concentration, i.e., the size of supercells, as well as the atomic relaxation, is crucial in predicting the magnetic coupling and electronic properties. As seen above, our findings help explain many puzzling experimental observations, notably the low saturation moments, the contracted lattice constants, and the highly insulating behavior. Our results also highlight the intrinsically complex nature of the spatial distribution and the magnetic structure of TM-doped DMS.

\section{ACKNOWLEDGMENTS}

We acknowledge the computing resources provided by the Australian Partnership for Advanced Computing (APAC) National Facility and by the Australian Centre for Advanced Computing and Communications (AC3). We thank the Australian Research Council for financial support. Work at Northwestern University Materials Research Center was supported by the NSF (through its MRSEC program). 
${ }^{1}$ H. Munekata, H. Ohno, S. von Molnar, A. Segmuller, L. L. Chang, and L. Esaki, Phys. Rev. Lett. 63, 1849 (1989).

${ }^{2}$ H. Ohno, A. Shen, F. Matsukura, A. Oiwa, A. Endo, S. Katsumoto, and Y. Iye, Appl. Phys. Lett. 69, 363 (1996).

${ }^{3}$ H. Ohno, Science 281, 951 (1998); S. A. Wolf, D. D. Awschalom, R. A. Buhrman, J. M. Daughton, S. von Molnar, M. L. Roukes, A. Y. Chtchelkanova, and D. M. Treger, ibid. 294, 1488 (2001); I. Zutic, J. Fabian, and S. D. Sarma, Rev. Mod. Phys. 76, 323 (2004).

${ }^{4}$ Spin Electronics, edited by M. Ziese and M. J. Thornton (Springer, Berlin, 2001).

${ }^{5}$ B. Beschoten, E. Johnston-Halperin, D. K. Young, M. Poggio, J. E. Grimaldi, S. Keller, S. P. DenBaars, U. K. Mishra, E. L. Hu, and D. D. Awschalom, Phys. Rev. B 63, 121202(R) (2001).

${ }^{6}$ S. Krishnamurthy, M. van Schilfgaarde, and N. Newman, Appl. Phys. Lett. 83, 1761 (2003).

${ }^{7}$ T. Dietl, H. Ohno, F. Matsukura, J. Cibert, and D. Ferrand, Science 287, 1019 (2000).

${ }^{8}$ T. Graf, M. Gjukic, M. S. Brandt, M. Stutzmann, and O. Ambacher, Appl. Phys. Lett. 81, 5159 (2002).

${ }^{9}$ R. Y. Korotkov, J. M. Gregie, and B. W. Wessels, Appl. Phys. Lett. 80, 1731 (2002).

${ }^{10}$ S. V. Novikov, K. W. Edmonds, A. D. Giddings, K. Y. Wang, C. R. Staddon, R. P. Campion, B. L. Gallagher, and C. T. Foxon, Semicond. Sci. Technol. 19, L13 (2004).

${ }^{11}$ K. Ando, Appl. Phys. Lett. 82, 100 (2003).

${ }^{12}$ M. E. Overberg, C. R. Abernathy, S. J. Pearton, N. A. Theodoropoulou, K. T. McCarthy, and A. F. Hebardet, Appl. Phys. Lett. 79, 1312 (2001).

${ }^{13}$ M. L. Reed, M. K. Ritums, H. H. Stadelmaier, M. J. Reed, C. A. Parker, S. M. Bedair, and N. A. El-Masry, Mater. Lett. 51, 500 (2001).

${ }^{14}$ N. Theodoropoulou, A. F. Hebard, M. E. Overberg, C. R. Abernathy, S. J. Pearton, S. N. G. Chu, and R. G. Wilson, Appl. Phys. Lett. 78, 3475 (2001).

${ }^{15}$ S. Sonada, S. Shimizu, T. Sasaki, Y. Yamamoto, and H. Hori, J. Cryst. Growth 237, 1358 (2002).

${ }^{16}$ T. Sasaki, S. Sonoda, Y. Yamamoto, K. Suga, S. Shimizu, K. Kindo, and H. Hori, J. Appl. Phys. 91, 7911 (2002).

${ }^{17}$ H. Hori, S. Sonoda, T. Sasaki, Y. Yamamoto, S. Shimizu, K. Suga, and K. Kindo, Physica B 324, 142 (2002).

${ }^{18}$ K. Sato and H. Katayama-Yoshida, Jpn. J. Appl. Phys., Part 2 40, L485 (2001).

${ }^{19}$ S. Dhar, O. Brandt, A. Trampert, K. J. Friedland, Y. J. Sun, and K. H. Ploog, Phys. Rev. B 67, 165205 (2003).

${ }^{20}$ M. Zajac, R. Doradzinski, J. Gosk, J. Szczytko, M. LefeldSosnowska, M. Kaminska, A. Twardowski, M. Palczewska, E. Grzanka, and W. Gebicki, Appl. Phys. Lett. 78, 1276 (2001).

${ }^{21}$ M. Zajac, J. Gosk, M. Kaminska, A. Twardowski, T. Szyszko, and S. Podsiadlo, Appl. Phys. Lett. 79, 2432 (2001).

${ }^{22}$ V. A. Guzenko, N. Thillosen, A. Dahmen, R. Calarco, Th. Schapers, L. Houben, M. Luysberg, B. Schineller, M. Heuken, and A. Kaluza, J. Appl. Phys. 96, 5663 (2004).

${ }^{23}$ H. Yang, H. Al-Brithen, A. R. Smith, J. A. Borchers, R. L. Cappelletti, and M. D. Vaudin, Appl. Phys. Lett. 78, 3860 (2001).

${ }^{24}$ S. Dhar, O. Brandt, A. Trampert, L. Daweritz, K. J. Friedland, K. H. Ploog, J. Keller, B. Beschoten, and G. Guntherodt, Appl. Phys. Lett. 82, 2077 (2003).

${ }^{25}$ K. H. Kim, K. J. Lee, D. J. Kim, H. J. Kim, Y. E. Ihm, D. Djayaprawira, M. Takaheashi, C. S. Kim, C. G. Kim, and S. H.
Yoo, Appl. Phys. Lett. 82, 1775 (2003).

${ }^{26}$ R. Frazier, G. T. Thaler, M. E. Overberg, B. Gila, C. R. Abernathy, and S. J. Pearton, Appl. Phys. Lett. 83, 1758 (2003).

${ }^{27}$ K. J. Lee, F. C. Yu, J. A. Kim, D. J. Kim, Y. H. Kang, H. J. Kim, and Y. E. Ihm, Phys. Status Solidi B 241, 1525 (2004).

${ }^{28}$ M. Zajac, J. Gosk, E. Grzanka, M. Kaminska, A. Twardowski, B. Strojek, T. Szyszko, and S. Podsiadlo, J. Appl. Phys. 93, 4715 (2001).

${ }^{29}$ Y. Shon, Y. H. Kwon, S. U. Yuldashev, J. H. Leem, C. S. Park, D. J. Fu, and X. J. Fan, Appl. Phys. Lett. 81, 1845 (2002).

${ }^{30}$ R. Giraud, S. Kuroda, S. Marcet, E. Bellet-Amalric, X. Biquard, B. Barbara, D. Fruchart, D. Ferrand, J. Cibert, and H. Mariette, Europhys. Lett. 65, 553 (2004).

${ }^{31}$ G. T. Thaler, M. E. Overberg, B. Gila, R. Frazier, C. R. Abernathy, S. J. Pearton, J. S. Lee, S. Y. Lee, Y. D. Park, Z. G. Khim, J. Kim, and F. Ren, Appl. Phys. Lett. 80, 3964 (2002).

${ }^{32}$ K. H. Kim, K. J. Lee, D. J. Kim, C. S. Kim, H. C. Lee, C. G. Kim, S. H. Yoo, H. J. Kim, and Y. E. Ihm, J. Appl. Phys. 93, 6793 (2003).

${ }^{33}$ E. Sarigiannidou, F. Wilhelm, E. Monroy, R. M. Galera, E. Bellet-Amalric, A. Rogalev, J. Goulon, J. Cibert, and H. Mariette, Phys. Rev. B 74, 041306(R) (2006).

${ }^{34}$ Y. Shon, Y. H. Kwon, D. Y. Kim, X. Fan, D. Fu, and T. W. Kang, Jpn. J. Appl. Phys., Part 1 40, 5304 (2001).

${ }^{35}$ L. M. Sandratskii, P. Bruno, and J. Kudrnovsky, Phys. Rev. B 69, 195203 (2004).

${ }^{36}$ Y.-J. Zhao, W. T. Geng, K. T. Park, and A. J. Freeman, Phys. Rev. B 64, 035207 (2001); M. Jain, L. Kronik, J. R. Chelikowsky, and V. V. Godlevsky, ibid. 64, 245205 (2001).

${ }^{37}$ B. Sanyal, O. Bengone, and S. Mirbt, Phys. Rev. B 68, 205210 (2003).

${ }^{38}$ S. H. Wei, X. G. Gong, G. M. Dalpian, and S. H. Wei, Phys. Rev. B 71, 144409 (2005).

${ }^{39}$ X. Luo and R. M. Martin, Phys. Rev. B 72, 035212 (2005).

${ }^{40}$ Z. S. Popovic, S. Satpathy, and W. C. Mitchel, Phys. Rev. B 70, 161308(R) (2004).

${ }^{41}$ E. Kulatov, H. Nakayama, H. Mariette, H. Ohta, and Y. A. Uspenskii, Phys. Rev. B 66, 045203 (2002).

${ }^{42}$ P. Mahadevan, J. M. Osorio-Guillen, and A. Zunger, Appl. Phys. Lett. 86, 172504 (2005).

${ }^{43}$ K. Sato, W. Schweika, P. H. Dederichs, and H. KatayamaYoshida, Phys. Rev. B 70, 201202(R) (2004).

${ }^{44}$ G. P. Das, B. K. Rao, and P. Jena, Phys. Rev. B 68, 035207 (2003); 69, 214422 (2004).

${ }^{45}$ J. Kang, K. J. Chang, and H. Katayama-Yoshida, J. Supercond. 18, 55 (2005).

${ }^{46}$ P. Mahadevan and S. Mahalakshmi, Phys. Rev. B 73, 153201 (2006).

${ }^{47}$ B. Sanyal and S. Mirbt, J. Magn. Magn. Mater. 290-291, 1408 (2005).

${ }^{48}$ Q. Wang, Q. Sun, P. Jena, and Y. Kawazoe, Phys. Rev. Lett. 93, 155501 (2004).

${ }^{49}$ X. Y. Cui, J. E. Medvedeva, B. Delley, A. J. Freeman, N. Newman, and C. Stampfl, Phys. Rev. Lett. 95, 256404 (2005).

${ }^{50}$ X. Y. Cui, J. E. Medvedeva, B. Delley, A. J. Freeman, and C. Stampfl, Phys. Rev. B 75, 155205 (2007).

${ }^{51}$ G. Martinez-Criado, A. Somogyi, M. Hermann, M. Eickhoff, and M. Stutzmann, Jpn. J. Appl. Phys., Part 2 43, L695 (2004).

${ }^{52}$ G. Martínez-Criado, A. Somogyi, A. Homs, R. Tucoulou, and J. Susini, Appl. Phys. Lett. 87, 061913 (2005). 
${ }^{53}$ G. Martínez-Criado, A. Somogyi, S. Ramos, J. Campo, R. Tucoulou, M. Salome, J. Susini, M. Hermann, M. Eickhoff, and M. Stutzmann, Appl. Phys. Lett. 86, 131927 (2005).

${ }^{54}$ M. van Schilfgaarde and O. N. Mryasov, Phys. Rev. B 63, 233205 (2001).

55 J. L. Xu, M. van Schilfgaarde, and G. D. Samolyuk, Phys. Rev. Lett. 94, 097201 (2005).

${ }^{56}$ B. K. Rao and P. Jena, Phys. Rev. Lett. 89, 185504 (2002).

${ }^{57}$ L. M. Sandratskii, P. Bruno, and S. Mirbt, Phys. Rev. B 71, 045210 (2005).

${ }^{58}$ T. Hynninen, H. Raebiger, J. von Boehm, and A. Ayuela, Appl. Phys. Lett. 88, 122501 (2006).

${ }^{59}$ A. Filippetti, N. A. Spaldin, and S. Sanvito, J. Magn. Magn. Mater. 290-291, 1391 (2005).

${ }^{60}$ M. H. Kane, A. Asghar, C. R. Vestal, M. Strassburg, J. Senawiratne, Z. J. Zhang, N. Dietz, C. J. Summers, and I. T. Ferguson, Semicond. Sci. Technol. 20, L5 (2005).

${ }^{61}$ T. Graf, M. Gjukic, M. Hermann, M. S. Brandt, M. Stutzmann, and O. Ambacher, Phys. Rev. B 67, 165215 (2003).

${ }^{62}$ S. Sonoda, I. Tanaka, H. Ikeno, T. Yamamoto, F. Oba, T. Araki, Y. Yamamoto, K. Suga, Y. Nanishi, Y. Akasaka, K. Kindo, and H. Hori, J. Phys.: Condens. Matter 18, 5615 (2006).

${ }^{63}$ D. J. Keavney, S. H. Cheung, S. T. King, M. Weinert, and L. Li, Phys. Rev. Lett. 95, 257201 (2005).

${ }^{64}$ J. P. Perdew, K. Burke, and M. Ernzerhof, Phys. Rev. Lett. 77, 3865 (1996).

${ }^{65}$ B. Delley, J. Chem. Phys. 113, 7756 (2000); 92, 508 (1990).

${ }^{66}$ B. Delley, Phys. Rev. B 66, 155125 (2002).

${ }^{67}$ C. Stampfl and C. G. Van de Walle, Phys. Rev. B 59, 5521 (1999), and references therein.

${ }^{68}$ C. G. Van de Walle, J. Appl. Phys. 95, 3851 (2004).

${ }^{69}$ J. F. Janak, Phys. Rev. B 18, 7165 (1978).

${ }^{70} \mathrm{We}$ use the total energy of nonmagnetic rocksalt $\mathrm{MnN}$ and add the energy difference between nonmagnetic and AFM $\mathrm{MnN}$ of $0.27 \mathrm{eV} / \mathrm{MnN}$ as obtained by W. R. L. Lambrecht, M. Prikhodko, and M. S. Miao, Phys. Rev. B 68, 174411 (2003).

${ }^{71}$ Our calculated magnetic moment for the FM state is $2.42 \mu_{B}$, which is in agreement with $2.45 \mu_{B}$ as reported by A. Sakuma, J. Magn. Magn. Mater. 187, 105 (1998).

${ }^{72}$ We use the total energy of body-centered-cubic (bcc) Mn plus the energy difference between bcc Mn and the ground state $\alpha$-Mn of $0.15 \mathrm{eV} / \mathrm{Mn}$ as obtained by D. Hobbs and J. Hafner, J. Phys.: Condens. Matter 13, L681 (2001).

${ }^{73}$ C. Stampfl and C. G. Van de Walle, Phys. Rev. B 65, 155212 (2002)

${ }^{74}$ S. Limpijumnong and C. G. Van de Walle, Phys. Rev. B 69, 035207 (2004).
${ }^{75}$ L. Kronik, M. Jain, and J. R. Chelikowsky, Phys. Rev. B 66, 041203(R) (2002).

${ }^{76}$ V. L. Moruzzi, Phys. Rev. Lett. 57, 2211 (1986).

${ }^{77}$ Y. L. Soo, G. Kioseoglou, S. Kim, S. Huang, Y. H. Kao, S. Kuwabara, S. Owa, T. Kondo, and H. Munekata, Appl. Phys. Lett. 79, 3926 (2001).

${ }^{78}$ M. Sato, H. Tanida, K. Kato, T. Sasaki, Y. Yamamoto, S. Sonoda, S. Shimizu, and H. Hori, Jpn. J. Appl. Phys., Part 1 41, 4513 (2002).

${ }^{79}$ G. T. Thaler, R. Frazier, B. Gila, J. Stapleton, M. Davidson, C. R. Abernathy, and S. J. Pearton, Appl. Phys. Lett. 84, 2578 (2004).

${ }^{80}$ X. Y. Cui, B. Delley, A. J. Freeman, and C. Stampfl, Phys. Rev. Lett. 97, 016402 (2006).

${ }^{81}$ G. M. Dalpian and S. H. Wei, J. Appl. Phys. 98, 083905 (2005).

${ }^{82}$ J. Kang and K. J. Chang, Physica B 376-377, 635 (2006).

${ }^{83}$ G. M. Dalpian, S. H. Wei, X. G. Gong, A. J. R. da Silva, and A. Fazzio, Solid State Commun. 138, 353 (2006).

${ }^{84}$ H. Raebiger, A. Ayuela, and J. von Boehm, Phys. Rev. B 72, 014465 (2005).

${ }^{85}$ P. Mahadevan and A. Zunger, Phys. Rev. B 68, 075202 (2003).

${ }^{86}$ R. K. Singh, S. Y. Wu, H. X. Liu, L. Gu, D. J. Smith, and N. Newman, Appl. Phys. Lett. 86, 012504 (2005).

${ }^{87}$ T. Takeuchi, Y. Harada, T. Tokushima, M. Taguchi, Y. Takata, A. Chainani, J. J. Kim, H. Makino, T. Yao, T. Yamamoto, T. Tsukamoto, S. Shin, and K. Kobayashi, Phys. Rev. B 70, 245323 (2004).

${ }^{88}$ P. Bobadova-Parvanova, K. A. Jackson, S. Srinivas, and M. Horoi, Phys. Rev. A 67, 061202(R) (2003).

${ }^{89}$ H. Amano, M. Kito, K. Hiramutsu, and I. Akasaki, Jpn. J. Appl. Phys., Part 2 28, L2112 (1989).

${ }^{90}$ I. Akasaki, H. Amano, Y. Koide, K. Hiramatsu, and N. Sawaki, J. Cryst. Growth 98, 209 (1989).

${ }^{91}$ L. Gu, S. Y. Wu, H. X. Liu, R. K. Singh, N. Newman, and D. J. Smith, J. Magn. Magn. Mater. 290-291, 1395 (2005).

${ }^{92}$ S. Granville, F. Budde, B. J. Ruck, H. J. Trodahl, G. V. M. Williams, A. Bittar, M. Byan, J. Kennedy, A. Markwitz, J. B. Metson, K. E. Prince, J. M. Cairney, and M. C. Ridgway, J. Appl. Phys. 100, 084310 (2006).

${ }^{93}$ S. Y. Wu and N. Newman, Appl. Phys. Lett. 89, 142105 (2006).

${ }^{94}$ R. Yang, H. Q. Yang, and A. R. Smith, Appl. Phys. Lett. 88, 173101 (2006).

${ }^{95}$ G. Thaler, R. Frazier, B. Gila, J. Stapleton, M. Davidson, C. R. Abernathy, S. J. Pearton, and C. Segre, Appl. Phys. Lett. 84, 1314 (2004).

${ }^{96}$ T. Fukushima, K. Sato, H. Katayama-Yoshida, and P. H. Dederichs, Jpn. J. Appl. Phys., Part 2 45, L416 (2006). 